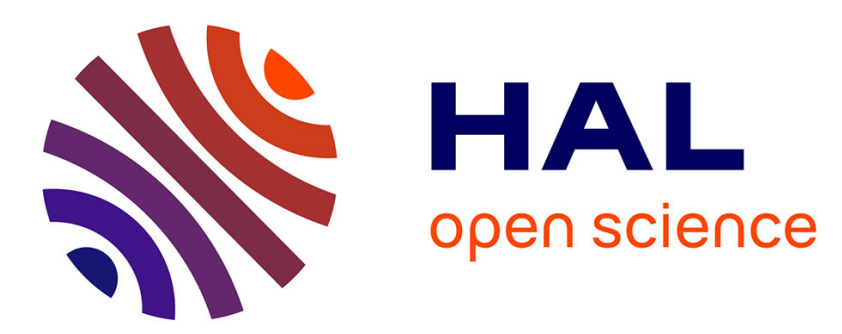

\title{
Surface-tethered polymers in polymeric matrices
}

Miguel Aubouy, Elie Raphaël

\section{To cite this version:}

Miguel Aubouy, Elie Raphaël. Surface-tethered polymers in polymeric matrices. Journal de Physique II, 1993, 3 (4), pp.443-448. 10.1051/jp2:1993142 . jpa-00247845

\section{HAL Id: jpa-00247845 https://hal.science/jpa-00247845}

Submitted on 1 Jan 1993

HAL is a multi-disciplinary open access archive for the deposit and dissemination of scientific research documents, whether they are published or not. The documents may come from teaching and research institutions in France or abroad, or from public or private research centers.
L'archive ouverte pluridisciplinaire HAL, est destinée au dépôt et à la diffusion de documents scientifiques de niveau recherche, publiés ou non, émanant des établissements d'enseignement et de recherche français ou étrangers, des laboratoires publics ou privés. 
Classification

Physics Abstracts

36.20

\title{
Short Communication
}

\section{Surface-tethered polymers in polymeric matrices}

\author{
Miguel Aubouy and Elie Raphaël \\ Laboratoire de Physique de la Matière Condensée, URA 792 CNRS, Collège de France, 11 Place \\ Marcelin-Berthelot, 75231 Patis Cedex 05, France
}

(Received 30 November 1992, accepted 29 January 1993)

\begin{abstract}
Recently Budkowski et al. used nuclear reaction analysis to study polymer brushes consisting of end-tethered deuterated polystyrene tails within a polystyrene homopolymer matrix. They measured the concentration-depth profile as a function of the surface coverage $\sigma$ of tails and the degree of polymerisation of the matrix. For the matrix with the largest degree of polymerisation, the variation of the effective brush thickness $L$ with $\sigma$ can be fitted by the apparent power law $L \propto \sigma^{q}$ with $q=0.54 \pm 0.06$. This scaling law is not expected theoretically for surface-tethered polymers exposed to a polymeric matrix consisting of chemically identical chains ( $\chi=0$, where $\chi$ is the interaction parameter). In the present note we would like to propose an explanation based on the fact that mixtures of deuterated and protonated polystyrene chains are characterized by a small (but non-zero) value of $\chi$.
\end{abstract}

\section{Introduction.}

Polymer chains tethered by one end to a flat solid surface at high enough coverage stretch away from the surface forming a polymer "brush". Being a central model in many important problems in polymer science, polymer brushes have been the subject of numerous theoretical and experimental studies [1] in the last 15 years. The pioneering work of Alexander and de Gennes [2, 3], based on scaling arguments, was followed more recently by computer simulations $[4,5]$ and self-consistent field calculations [6-9]. Most of the existing experimental studies focus on polymer brushes exposed to a low molecular weight solvent and much less is known of the behavior of surface-tethered polymers in polymeric matrices. Very recently, Budkowski et al. [10] studied, using nuclear reaction analysis, polymer brushes consisting of end-tethered deuterated polystyrene tails ( $N$ monomers per tail) in a polystyrene homopolymer matrix. They measured the concentration-depth profiles as a function of the surface coverage $\sigma$ of tails and the degree of polymerization $P$ of the polymer matrix. For the most part, the experimental result for the variation of the brush thickness $L$ with $\sigma$ and $P$ are consistent with scaling and 
mean-field models. The variation of $L$ with $\sigma$ for the highest $P$ matrix used in the experiment is however very intriguing: for $\sigma>4 \times 10^{-3}$, the data can be fitted by the apparent power law

$$
L \propto \sigma^{q} \quad q=0.54 \pm 0.06
$$

This scaling law is not expected theoretically for surface-tethered polymers exposed to a polymeric matrix consisting of chemically identical chains $(\chi=0$, where $\chi$ is the interaction parameter). In order to solve this apparent contradiction, the authors, who implicitly assumed a zero interaction parameter, suggested that the power law (1) may indicate a cross over region between the predicted regimes $L \propto \sigma^{0}$ and $L \propto \sigma^{1}$ (see Sect. 2) in the present letter we would like to propose another possible explanation based on the fact that mixtures of deuterated and protonated polystyrene chains are characterized by a small (but non zero) value of $\chi$, as shown by Bates and co-workers [11]. The paper is organized as follows: we first recall the brush conformation in the case of a chemically indentical polymer matrix (Sect. 2). We then consider the effect of a small chemical mismatch (Sect. 3). The paper ends with a short discussion.

Throughout this paper we adopt the Alexander-de Gennes scaling picture [2, 3]; in particular, we assume a steplike concentration profile and impose that all free ends be at the same distance from the surface.

\section{The chemically identical case $(\chi=0)$.}

Consider a brush made of chains, with degree of polymerization $N$, terminally grafted onto a flat surface and exposed to a solvent made of chemically identical chains, with degree of polymerization $P[3]$. The number of terminally grafted chains per unit area is $\sigma a^{-2}$, where $a$ is the monomer size. The average distance between two grafting sites is given by $D=a \sigma^{-1 / 2}$.

At sufficiently low $\sigma$, the grafted polymers do not overlap (the so-called mushroom regime [3]). The brush thickness $L$ is given by $L \cong a N^{1 / 2}$ for $P>N^{1 / 2}$ and $L \cong a N^{3 / 5} P^{-1 / 5}$ for $P<N^{1 / 2}$ (see regions (1) and (2) in Fig.1).

As $\sigma$ increases, the different chains begin to overlap for $D \cong L$. This defines an overlap concentration: $\sigma_{\mathrm{OV}} \cong N^{-1}$ for $P>N^{1 / 2}$ and $\sigma_{\mathrm{OV}} \cong P^{2 / 5} N^{-6 / 5}$ for $P<N^{1 / 2}$ In a Flory type approach, the free energy per chain is then given by:

$$
\frac{F}{k T} \cong \frac{L^{2}}{a^{2} N}+\frac{a^{3}}{P} \frac{N^{2}}{L D^{2}}+\frac{a^{6}}{P} \frac{N^{3}}{\left(L D^{2}\right)^{2}}
$$

The second term in equation (2) corresponds to two-body interactions while the third term corresponds to three-body interactions (we omit higher order interactions). These two terms correspond to an osmotic contribution. The origin of the screening factors $P^{-1}$ in equation (2) is explained in appendix 2. The first term in equation (2) represents the elastic contribution.

From equation (2) one can easily construct the $(P, \sigma)$ diagram represented in figure 1 (for more details see [15]):

- in region (3), two body interactions dominate the osmotic contribution and the brush thickness is given by: $L \cong a N P^{-1 / 3} \sigma^{1 / 3}$

- in region (4), repulsive interactions are not sufficient to swell the brush and the structure remains Gaussian: $L \cong a N^{1 / 2}$

At higher $\sigma$, we reach region (6) where the $P$ chains are almost completely expelled from the brush: the brush is "dry". In this region the volume fraction of the grafted polymer is of order unity and $L \cong a N \sigma$. 


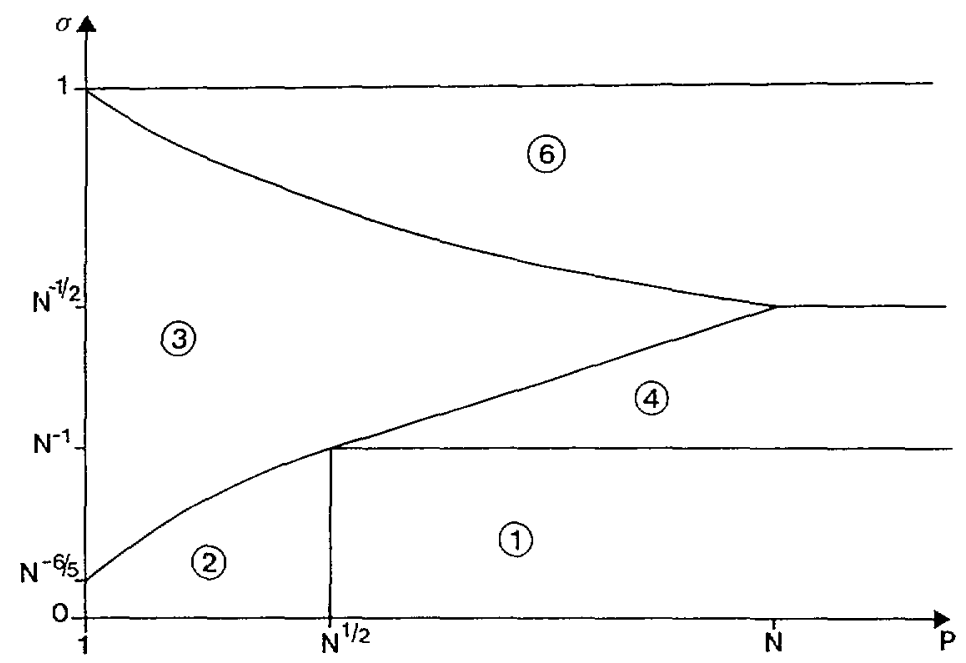

Fig. 1. - Schematic $(P, \sigma)$ diagram for a brush exposed to a chemically identical $(\chi=0)$ high molecular weight solvent. The thickness of the brush in the different regions is given by: (1): $L \cong$ $a N^{1 / 2} ;(2): L \cong a N^{3 / 5} P^{-1 / 5} ;(3): L \cong a N P^{-1 / 3} \sigma^{1 / 3} ;(4): L \cong a N^{1 / 2} ;(6): L \cong a N \sigma$. The cross over between the different regions are given in the appendix 2 .

\section{Influence of a small chemical difference $(\chi>0)$.}

We now consider the less familiar case of small chemical mismatch between the grafted polymer and the polymer matrix. This case was first considered by Zhulina and Borisov [16]. The chemical mismatch is expressed in terms of a non-zero interaction parameter $\chi$, which generates a new term in the Flory free energy:

$$
\frac{F}{k T} \cong \frac{L^{2}}{a^{2} N}+\frac{a^{3}}{P}(1-2 P \chi) \frac{N^{2}}{L D^{2}}+\frac{a^{6}}{P} \frac{N^{3}}{\left(L D^{2}\right)^{2}}
$$

We assume both the interaction parameter $\chi$ and the second virial coefficient $(1-2 P \chi) / P$ to be positive.

The overall shape of the $(P, \sigma)$ diagram is not drastically affected by this change as long as the three-body interactions are negligeable. Indeed, at low $\sigma$, we can build the $(P, \sigma)$ diagram figure 2 by simply considering the substitution $P \leftrightarrow P /(1-2 P \chi)$.

However, if we increase $\sigma$, we reach a region $\left(5^{\prime}\right)$ where three-body interactions become the dominant term in the osmotic contribution and the previous substitution ceases to be valid. In this region the free energy is given by:

$$
\frac{F}{k T} \cong \frac{L^{2}}{a^{2} N}+\frac{a^{6}}{P} \frac{N^{3}}{\left(L D^{2}\right)^{2}}
$$

Minimizing equation (4) leads to:

$$
L \cong a N P^{-1 / 4} \sigma^{1 / 2}
$$




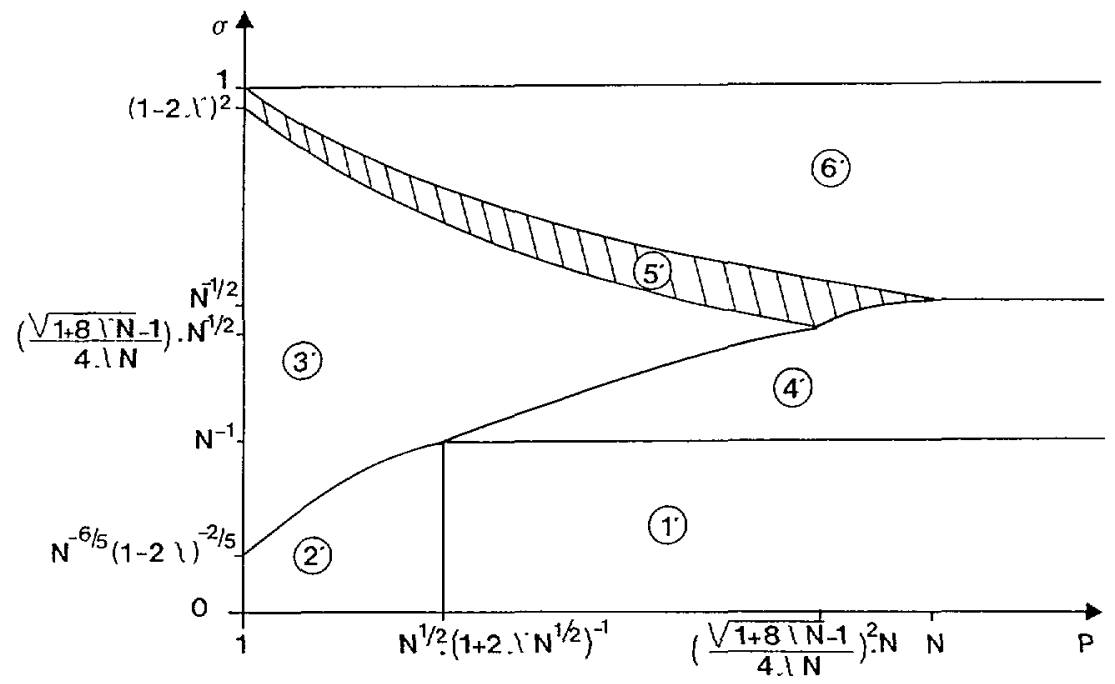

Fig. 2. - Schematic $(P, \sigma)$ diagram for a brush exposed to a high molecular weight solvent in the case of a small chemical mismatch. The thickness of the brush in the different regions is given by: $\left(1^{\prime}\right): L \cong a N^{1 / 2} ;\left(2^{\prime}\right): L \cong a N^{3 / 5} P^{-1 / 5}(1-2 P \chi)^{1 / 5} ;\left(3^{\prime}\right): L \cong a N P^{-1 / 3} \sigma^{1 / 3}(1-2 P \chi)^{1 / 3} ;\left(4^{\prime}\right):$ $L \cong a N^{1 / 2} ;\left(5^{\prime}\right): L \cong a N P^{1 / 4} \sigma^{1 / 2} ;\left(6^{\prime}\right): L \cong a N \sigma$. The cross over between the different regions are given in the appendix 2 .

The lower limit of the region $\left(5^{\prime}\right)$ arises when two-body and three-body interactions are comparable:

$$
\sigma_{\mathrm{CO}} \cong \frac{(1-2 P \chi)^{2}}{P^{1 / 2}}
$$

(where the subscript CO stands for cross over). The upper limit of this region is obtained by considering $\phi$, the volume fraction of grafted chains. From equation (5) we obtain $\phi \cong P^{1 / 4} \sigma^{1 / 2}$, and when $\phi=1$ we reach the dry brush region:

$$
\sigma_{\mathrm{CO}} \cong \frac{1}{P^{1 / 2}}
$$

If we increase $P$, we reach region $\left(4^{\prime}\right)$ where repulsive interactions do not affect the conformation of the brush. In region (5') the perturbation parameter is $\xi \equiv a^{6} N^{3} / P\left(R_{0}^{2} D^{2}\right)^{2}$ Setting $\xi \cong 1$ gives us the thrird limit of region $\left(5^{\prime}\right)$ :

$$
\sigma_{\mathrm{CO}} \cong \frac{P^{1 / 2}}{N}
$$

Since $\chi \neq 0$, the cross over equations (6) and (7) are different and define a new region ( $\left.5^{\prime}\right)$. A small chemical difference can thus drasticaly affect the $(P, \sigma)$ diagram by generating a new region (region $\left(5^{\prime}\right)$ ). In this region, some $P$ chains are still present in the brush and the osmotic contribution is dominated by three-body interactions; this leads to $L \propto \sigma^{1 / 2}$ Note that this region vanishes for $P>N$. 


\section{Concluding remarks.}

As shown in section 3, a small chemical mismatch is able to bring about a new region in the $(P, \sigma)$ diagram where the brush thickness scales as $\sigma^{1 / 2}$. This might provide an explanation for the apparent power law equation (1) observed by Budkowski et al. [10]. Moreover, the experimental results indicate that some $P$ chains are still present in the brush, in agreement with our predictions. From the study of Bates and coworkers we know that the experimental interaction parameter $\chi$ for a binary mixture of fully deuterated and protonated polystyrenes behaves as $\chi=(0.20 \pm 0.01) T^{-1}-(2.9 \pm 0.4) \times 10^{-4}$ where $T$ is the temperature [11, 17]. In the experimental conditions of Budkowski et al. we expect $\chi \cong 1.1 \times 10^{-4}$; this value enables us to check that the experiment of the reference [10] take place within the correct range of $\sigma$ values. We are aware of the fact that in the experiment of Budkowski et al. the length of the chains in the matrix is greater than the length of the tails $(P \cong 3 N)$ and, strictly speaking, we should not expect $L \propto \sigma^{1 / 2}$. Nevertheless, we think that our explanation remains plausible since, in our Flory type approach, all numerical factors are undetermined while they could well be relevant in the interpretation of the experiment.

\section{Acknowledgements.}

We would like to thank Françoise Brochard-Wyart and Jacob Klein for very useful comments.

\section{Appendix 1}

Consider an isolated linear chain, with degree of polymerization $N$, dissolved in a melt of shorter chains (degree of polymerization $P<N$ ). The monomer-monomer interactions of the $N$-chain are screened out by the $P$-chains. As a consequence, the bare excluded-volume parameter $v_{0}=a^{3}(1-2 \chi)$ is replaced by the effective parameter $v=a^{3}\left(P^{-1}-2 \chi\right)$. This result was first stated by Flory [12], but its physical interpretation in terms of screening is due to Edwards [13]. It can be simply deduced from the Flory-Huggins free energy of polymer mixtures [14]:

$$
\frac{F_{\text {site }}}{k T}=\frac{\varphi_{N}}{N} \operatorname{Ln} \varphi_{N}+\frac{1-f_{N}}{P} \operatorname{Ln}\left(1-\varphi_{N}\right)+\chi \varphi_{N}\left(1-\varphi_{N}\right)
$$

where $\varphi_{N}$ is the volume fraction of the $N$ monomers. In the limit of small $\varphi_{N}$, equation (9) leads to

$$
\frac{F_{\text {site }}}{k T}=\frac{\varphi_{N}}{N} \operatorname{Ln} \varphi_{N}+\frac{1}{2}\left(\frac{1}{P}-2 \chi\right) \varphi_{N}^{2}+\frac{1}{6 P} \varphi_{N}^{3}+
$$

Equation (10) shows that second virial coefficient (i.e. the excluded-volume parameter) is indeed given by $a^{3}\left(P^{-1}-2 \chi\right)$. It also indicates that the third virial coefficient is equal to $a^{6} / P$. Hence equations (2) and (3) for $\chi=0$ and $\chi \neq 0$ respectively.

\section{Appendix 2}

We give here the cross over between the differents regions of the $(P, \sigma)$ diagram: Diagram of figure $1:(1) /(2): P_{\mathrm{CO}} \equiv N^{1 / 2} ;(2) /(3): P^{2 / 5} N^{-6 / 5} ;(1) /(4): \sigma_{\mathrm{CO}} \equiv N^{-1} ;(3) /(4)$ : $\sigma_{\mathrm{CO}} \equiv P N^{-3 / 2} ;(3) /(6): \sigma_{\mathrm{CO}} \equiv P^{-1 / 2} ;(4) /(6): \sigma_{\mathrm{CO}} \equiv N^{-1 / 2}$; 
Diagram of figure 2: $\left(1^{\prime}\right) /\left(2^{\prime}\right) P_{\mathrm{CO}} \equiv N^{1 / 2}\left(1+2 N^{1 / 2} \chi\right)^{-1} ;\left(2^{\prime}\right) /\left(3^{\prime}\right) \sigma_{\mathrm{CO}} \equiv P^{2 / 5} N^{-6 / 5}(1-$ $2 P \chi)^{-2 / 5} ;\left(1^{\prime}\right) /\left(4^{\prime}\right) \sigma_{\mathrm{CO}} \equiv N^{-1} ;\left(3^{\prime}\right) /\left(4^{\prime}\right) \sigma_{\mathrm{CO}} \equiv P N^{-3 / 2}(1-2 P \chi)^{-1} ;\left(3^{\prime}\right) /\left(5^{\prime}\right) \sigma_{\mathrm{CO}} \equiv(1-$ $2 P \chi)^{2} P^{-1 / 2} ;\left(4^{\prime}\right) /\left(5^{\prime}\right) \sigma_{\mathrm{CO}} \equiv P^{1 / 2} N^{-1} ;\left(4^{\prime}\right) /\left(6^{\prime}\right) \sigma_{\mathrm{CO}} \equiv N^{-1 / 2} ;\left(5^{\prime}\right) /\left(6^{\prime}\right) \sigma_{\mathrm{CO}} \equiv P^{-1 / 2}$

\section{References}

[1] Recent reviews of this work can be found in HALPERIN A., TIRREL M. and LODGE T.P., Adv. Polym. Sci. 100 (1991) 31;

and MILNER S.T. Science 251 (1991).

[2] ALEXANDER S., J. Phys. France 38 (1977) 977.

[3] de GENNES P.-G., J. Phys. France 37 (1976) 1443; Macromolecules 13 (1980) 1069.

See also LEIBLER L., Makromol. Chem., Macromol. Symp. 16 (1988) 1.

[4] MURAT M., GREST G.S., Phys. Rev. Lett. 63 (1989) 1074; Macromolecules 22 (1991) 4054; Macromolecules 24 (1980) 704.

[5] LAI P.-Y., BINDER K., J. Chem. Phys. 95 (1991) 9299.

[6] HIRZ S.J., Modeling of Interactions Between Adsorbed Block Copolymer, M.S. Thesis, University of Minnesota, Minneapolis, MN, (1988).

[7] MILNER S.T., WITTEN T.A., CATES M.E., Europhys. Lett. 5 (1988) 413.

[8] MILNER S.T., WITTEN T.A., CATES M.E., Macromolecules 21 (1988) 2610.

[9] ZHULINA E.B., PRYAMITSYN V.A., BORISOV O.V., Vysokomol. Soedin. Ser. A 31 (1889) 185 ;

ZHULINA E.B., BORISOV O.V., PRYAMITSYN V.A., J. Colloid Interface Sci. 137 (1990) 495.

[10] BUDKOWSKI A., STEINER U., KLEIN J., FETTERS L.J., Europhys. Lett. 20 (1992) 499.

[11] BATES F.S., WIGNALL G.D., Phys. Rev. Lett. 57 (1991) 1429.

[12] FLORY P.J., J. Chem. Phys. 17 (1949) 303.

[13] EDWARDS S.F., Proc. Phys. Soc. 88 (1969) 265.

[14] FLORY P.J., Principle of Polymer Chemistry (Cornell University Press, Ithaca, NY, 1971), Chapter XII;

HUGGins M.J., J. Am. Chem. Soc. 64 (1942) 1972.

[15] RAPHAËL E., PINCUS P., FREDERICKSON G.H., Macromolecule in press.

[16] ZHULINA E.B., BORISOV O.V., J. Colloid Interface Sci 144 (1991) 507.

[17] Very recent experiments by Klein and coworkers revealed a weak compositional dependence of $\chi$, see BUDKOWSKI A., STEINER U., KLEIN J., SCHATZ G., Europhys. Lett. 18 (1992) 705. 